MATHEMATICS OF COMPUTATION

Volume 66, Number 220, October 1997, Pages 1709-1717

S $0025-5718(97) 00864-8$

\title{
FURTHER TABULATION OF THE ERDÖS-SELFRIDGE FUNCTION
}

\author{
RICHARD F. LUKES, RENATE SCHEIDLER, AND HUGH C. WILLIAMS
}

\begin{abstract}
For a positive integer $k$, the Erdös-Selfridge function is the least integer $g(k)>k+1$ such that all prime factors of $\left(\begin{array}{c}g(k) \\ k\end{array}\right)$ exceed $k$. This paper describes a rapid method of tabulating $g(k)$ using VLSI based sieving hardware. We investigate the number of admissible residues for each modulus in the underlying sieving problem and relate this number to the size of $g(k)$. A table of values of $g(k)$ for $135 \leq k \leq 200$ is provided.
\end{abstract}

\section{INTRODUCTION}

For $k \geq 1$, denote by $g(k)$ the least integer $>k+1$ such that no prime $p \leq k$ divides $\left(\begin{array}{c}g(k) \\ k\end{array}\right)$. This function grows rapidly with increasing $k$ and is consequently difficult to compute for even modest values of $k$. The behavior of $g(k)$ was first studied by Ecklund, Erdös and Selfridge [2] who tabulated $g(k)$ for $k \leq 40$ as well as $g(42), g(46)$, and $g(52)$. These are all the values of $g(k) \leq 2500000$ when $k \leq 100$. The table was extended to include all the values of $g(k)$ for $k \leq 140$ by Scheidler and Williams [1] using sieving techniques. The largest of these values, $g(139)$, is a 17 digit number. Sieving was continued for $141 \leq k \leq 155$ but the results were never published.

A number of lower bounds on $g(k)$ were proved and conjectured in [2] and by Erdös, Lacampagne and Selfridge in [3]. The best lower bound was recently established by Granville and Ramaré [5] who proved that there exists an absolute positive constant $c$ such that

$$
g(k)>\exp \left(c\left(\log ^{3} k / \log \log k\right)^{\frac{1}{2}}\right) .
$$

This implies that $g(k)$ grows faster than any polynomial in $k$.

This paper further extends computations and provides values of $g(k)$ for $135 \leq$ $k \leq 200$. We also repeated earlier tabulations and found an error in the value of $g(138)$ given in [1]. The computation was performed on the Manitoba Scalable Sieve Unit (MSSU), a very fast VLSI based sieving device developed by Lukes, Patterson and Williams [4]. We used a modification of the algorithm given in [1]. To make this paper somewhat self-contained, we begin with a brief review of the basics of sieving as well as the sieving method used in our computations. We analyze the number of admissible residues of the sieving problem arising from $g(k)$ in Section 3. Section 4 compares the size of the sieving problem for $g(k)$ with the actual value

Received by the editor October 18, 1994 and, in revised form, October 9, 1995 and August 21, 1996.

1991 Mathematics Subject Classification. Primary 11N25, 11Y70, 11-04.

The third author's research is supported by NSERC of Canada grant A7649.

(C)1997 American Mathematical Society 
of $g(k)$ and investigates gaps between $g(q-1)$ and $g(q)$ where $q$ is a prime. Our implementation on MSSU is discussed in Section 5. The paper concludes with a table of values of $g(k)(135 \leq k \leq 200)$.

\section{The SIEving ALGORITHM}

In order to solve a sieving problem, it is required to find solutions to a system of simultaneous linear congruences. More exactly, one needs to search for integers $x$ such that

$$
x \quad\left(\bmod m_{i}\right) \in R_{i} \quad \text { for } \quad i=1,2, \ldots, h,
$$

where $h$ is a positive integer, the moduli $m_{1}, m_{2}, \ldots, m_{h}$ are positive integers assumed to be pairwise relatively prime, and each set $R_{i}=\left\{r_{i 1}, r_{i 2}, \ldots, r_{i n_{i}}\right\}$, called the set of admissible residues for modulus $m_{i}$, consists of nonnegative integers less than $m_{i}(i=1,2, \ldots, h)$. Boundary conditions are placed on $x$, i. e. we may require $x$ to lie in a certain specified range, or we might wish to obtain the least solution of (2.1) that exceeds a fixed lower bound. Additional restrictions, checked by a filter, may be placed on $x$.

Over the past 75 years, a number of mechanical as well as computer based machines for solving sieving problems have been constructed (see [4] for a history of these machines). MSSU is the most recent and by far the fastest such device.

Our method for tabulating $g(k)$ as well as some of the results in Section 3 are derived from Kummer's well-known result that the binomial coefficient $\left(\begin{array}{l}n \\ k\end{array}\right)$ is relatively prime to a prime $p$ if and only if there are no "carries" when $k$ and $n-k$ are added in base $p$ (see [6, p. 220]). Since the sieving algorithm is described in detail in [1], we merely sketch it here. Let

$$
k=\sum_{i=0}^{m} a_{i} p^{i}, \quad 0 \leq a_{i} \leq p-1 \quad \text { for } \quad i=0,1, \ldots, m ; \quad a_{m} \neq 0
$$

be the base $p$ representation of $k$. (It is easy to compute the coefficients $a_{i}$, see formula (2.1) in [1]). For $i=0,1, \ldots, m$, set

$$
C_{i}=\left\{a_{i}, a_{i}+1, \ldots, p-1\right\}
$$

and recursively define the sets

$$
B_{0}=C_{0}, \quad B_{i}=B_{i-1}+C_{i} p^{i}=\left\{b+c p^{i} \mid b \in B_{i-1}, c \in C_{i}\right\} \quad(i=1,2, \ldots, m) .
$$

Then $B_{i}=C_{0}+C_{1} p+C_{2} p^{2}+\cdots+C_{i} p^{i}$ for $i=0,1, \ldots, m$, and $g(k)$ is the smallest integer $n \geq k+2$ such that

$$
n \quad\left(\bmod p^{m+1}\right) \in B_{m}
$$

or equivalently,

$$
n \quad\left(\bmod p^{m}\right) \in B_{m-1}
$$

and

$$
\left\lfloor\frac{n}{p^{m}}\right\rfloor \quad(\bmod p) \geq a_{m} .
$$

To compute $g(k)$ for fixed $k$, the moduli $m_{i}$ in $(2.1)$ are the values $p^{m}$ where $p$ is a prime, $p \leq k$, and $p^{m} \leq k<p^{m+1}$, i. e. $m=\left\lfloor\log _{p} k\right\rfloor$. For each modulus $p^{m}$, the corresponding set of admissible residues is $B_{m-1}$. Each solution $n$ of (2.3) is 
checked for filter condition (2.4). The least integer $n \geq k+2$ satisfying both (2.3) and (2.4) for all primes $p \leq k$ is $g(k)$.

\section{Number OF ADMissible RESIDUES}

Let $p \leq k$ be a fixed prime and set $m=\left\lfloor\log _{p} k\right\rfloor$. Then each set $C_{i}$ contains $\left|C_{i}\right|=p-a_{i}$ elements $(i=0,1, \ldots, m)$, so for $i \neq j$, we have $\left|C_{i} p^{i}+C_{j} p^{j}\right|=$ $\left(p-a_{i}\right)\left(p-a_{j}\right)$. Hence, the number of residues for each modulus is given as follows.

Lemma 3.1. The number of admissible residues for modulus $p^{m}$ is

$$
r_{p}=\left|B_{m-1}\right|=\prod_{i=0}^{m-1}\left(p-a_{i}\right) .
$$

If the modulus is a prime, i. e. $m=1$, then the base $p$ representation of $k$ is $k=a_{1} p+a_{0}$, so $p-a_{0}=\left(a_{1}+1\right) p-k$. Hence in this case, we have

Corollary 3.2. If the modulus is a prime $p$, then $p$ divides $k+r_{p}$.

Clearly, the number of residues $r_{p}$ for modulus $p^{m}$ is between 1 and $p^{m}$, inclusive. If the number of admissible residues is maximal, i. e. $r_{p}=p^{m}$, then (2.3) is always satisfied and we do not need to include modulus $p^{m}$ in the congruences. It is easy to establish the exact form of $k$ in the extreme cases $r_{p}=p^{m}$ and $r_{p}=1$. For single residue congruences, we can also determine the unique residue.

Lemma 3.3. $r_{p}=p^{m}$ if and only if $k=a p^{m}$ where $1 \leq a \leq p-1$.

Proof. By Lemma 3.1, $r_{p}=p^{m}$ if and only if $a_{i}=0$ for $i=0,1, \ldots m-1$, so $k=a_{m} p^{m}, 1 \leq a_{m} \leq p-1$.

Lemma 3.4. $r_{p}=1$ if and only if $k=a p^{m}-1$ where $2 \leq a \leq p$.

Proof. By Lemma 3.1, $r_{p}=1$ if and only if $a_{i}=p-1$ for $i=0,1, \ldots, m-1$, so

$$
k=a_{m} p^{m}+\sum_{i=0}^{m-1}(p-1) p^{i}=a_{m} p^{m}+p^{m}-1=\left(a_{m}+1\right) p^{m}-1,
$$

$1 \leq a_{m}+1 \leq p$.

Lemma 3.5. If $r_{p}=1$, then the residue corresponding to modulus $p^{m}$ is $p^{m}-1$.

Proof. As in the previous lemma, if $r_{p}=1$, then $a_{i}=p-1$ for $i=0,1, \ldots m-1$, i. e. $B_{m-1}$ contains only the residue $\sum_{i=0}^{m-1}(p-1) p^{i}=p^{m}-1$.

We now compare the number of admissible residues for the two consecutive values $g(k-1)$ and $g(k)$ in the special case where $k$ is a prime power. Let $k=q^{t}$ where $q$ is a prime and $t \geq 1$. As before, let $p \leq k$ be a fixed prime and set $m=\left\lfloor\log _{p} k\right\rfloor$. To distinguish between quantities pertaining to different $k$ values, we include $k$ as an argument, i. e. write $r_{p}(k), C_{i}(k)$ etc.

Case 1: $p=q$. Then $m=t, k=p^{m}$ and by Lemma 3.3, $r_{p}(k)=p^{m}$. Now $k-1=$ $p^{m}-1$, so if $m>1$, then $k-1=p \cdot p^{m-1}-1$ and $r_{p}(k-1)=1$ by Lemma 3.4 (here, the corresponding modulus is $p^{m-1}$ ). If $m=1$, then $k-1=p-1<p$, so the moduli used in searching for $g(k-1)$ do not include a power of $p$. 
Case 2: $p \neq q$. Then it is easy to see that $\left\lfloor\log _{p}(k-1)\right\rfloor=m$. Let the $p$-ary representation of $k-1$ be

$$
k-1=\sum_{i=0}^{m} a_{i} p^{i}, \quad 0 \leq a_{i} \leq p-1 \quad \text { for } \quad i=0,1, \ldots, m ; \quad a_{m} \neq 0 .
$$

Since $p$ does not divide $k=q^{t}$, we must have $a_{0} \neq p-1$, so the $p$-ary representation of $k$ is

$$
k=\sum_{i=1}^{m} a_{i} p^{i}+\left(a_{0}+1\right) .
$$

Hence $C_{0}(k)=C_{0}(k-1) \backslash\left\{a_{0}\right\}$ and $C_{i}(k)=C_{i}(k-1)$ for $i=1,2, \ldots m$. It follows that

$$
\begin{aligned}
r_{p}(k) & =\left(p-a_{0}-1\right) \prod_{i=1}^{m-1}\left(p-a_{i}\right)=r_{p}(k-1)-\prod_{i=1}^{m-1}\left(p-a_{i}\right) \\
& =r_{p}(k-1)\left(1-\frac{1}{p-a_{0}}\right) .
\end{aligned}
$$

In summary:

Lemma 3.6. Let $k=q^{t}, q$ a prime, $t \geq 1$. Then for any prime $p \leq k$, the number of admissible residues for modulus $p^{m}, m=\left\lfloor\log _{p} k\right\rfloor$, satisfies the following properties.

1. If $p=q$, then $r_{p}(k)=p^{m}, r_{p}(k-1)=1$ if $m>1$, and no power of $p$ is included in the moduli for $k-1$ if $m=1$.

2. If $p \neq q$, then

$$
r_{p}(k)=r_{p}(k-1)\left(1-\frac{1}{p-k_{p}}\right),
$$

where $k_{p} \equiv k-1(\bmod p), 1 \leq k_{p} \leq p-1$.

Corollary 3.7. Let $k=q$ be a prime. Then the moduli in the congruences for both $g(q)$ and $g(q-1)$ are exactly the powers $p^{m}$ where $p$ is a prime less than $q$ and $m=\left\lfloor\log _{p} k\right\rfloor$. Furthermore, for each such prime $p$,

$$
r_{p}(q)=r_{p}(q-1)\left(1-\frac{1}{p-q_{p}}\right)
$$

where $q_{p} \equiv q-1(\bmod p), 1 \leq q_{p} \leq p-1$.

We conclude this section with a brief analysis of the filter conditions for both $k$ and $k-1$ when $k=q$ is a prime.

Lemma 3.8. If $k=q$ is a prime, then the filter condition (2.4) is satisfied for any solution candidate $n$ for either $g(q)$ or $g(q-1)$.

Proof. Let $p \leq q$ be a prime. Since each solution candidate $n$ for either $g(q)$ or $g(q-1)$ satisfies $n \geq(q-1)+2=q+1>p$, the left-hand side of (2.4) is always at least 1 . If $p<q$, then the base $p$ representations of $q$ and $q-1$, respectively, are $q=p+(q-p)$ and $q-1=p+(q-p-1)$, so in either case $a_{m}=a_{1}=1$ and (2.4) always holds. If $p=q$, then the prime $p$ is not included in the filter condition for $k=q-1$, and for $k=q$, we have again $a_{m}=a_{1}=1$, so (2.4) is always satisfied. 


\section{Size OF $g(k)$}

Since computations show that there are large variations in the size of $g(k)$ for different values of $k$, we thought it useful to correlate for given $k$ the probability that a solution candidate $n$ passes the sieve and filter for $g(k)$ with the actual value of $g(k)$. For a prime $p \leq k$, denote by $P_{p}(k)$ the probability that a solution candidate $n$ satisfies (2.2). Then under the reasonable assumption that each residue in $B_{m}=B_{m}(k)$ is equally likely, we get

$$
P_{p}(k)=\frac{\left|B_{m}(k)\right|}{p^{m+1}},
$$

so the probability that $n$ satisfies $(2.2)$ for all primes $p \leq k$ is

$$
P(k)=\prod_{p \leq k} P_{p}(k)=\prod_{p \leq k} \frac{\left|B_{m}(k)\right|}{p^{m+1}} .
$$

Since $P(k)$ determines the size of the range that needs to be sieved before $g(k)$ is found, with a larger value of $P(k)$ resulting in a smaller sieving range, we expect that $P(k)$ is approximately inverse proportional to $g(k)$. We therefore computed the product $R(k)=P(k) g(k)$ for $150 \leq k \leq 200$. The following table gives a sequence of ranges for $R(k)$ and indicates for each range the number of values of $k$ for which $R(k)$ fell within that range.

\begin{tabular}{|c|c|}
\hline Range & Number of $R(k)$ in Range \\
\hline$<0.1$ & 4 \\
$0.1-0.5$ & 23 \\
$0.5-1.0$ & 6 \\
$1.0-1.5$ & 7 \\
$1.5-2.0$ & 5 \\
$2.0-2.5$ & 2 \\
$2.5-3.0$ & 2 \\
$3.0-3.5$ & 0 \\
$3.5-4.0$ & 0 \\
$>4.0$ & 2 \\
\hline
\end{tabular}

Note that almost all values of $R(k)$ are between 0.1 and 2 , and that $0.1 \leq R(k)<$ 0.5 for nearly half the values of $k$. Only six values of $k$ produced extreme values of $R(k)$ which are given below.

\begin{tabular}{|c|c|}
\hline$k$ & $R(k)$ \\
\hline 199 & 0.011 \\
174 & 0.044 \\
162 & 0.048 \\
196 & 0.053 \\
\hline
\end{tabular}

\begin{tabular}{|c|c|}
\hline$k$ & $R(k)$ \\
\hline 186 & 4.411 \\
153 & 4.430 \\
& \\
& \\
\hline
\end{tabular}

Our table of values of $g(k)$ also shows that there are frequently significant gaps in size between $g(q-1)$ and $g(q)$ where $q$ is a prime. Let $p<q$ be a prime. By 
Lemma 3.8, we can ignore the filter condition (2.4), so from (2.3), we obtain

$$
P_{p}(k)=\frac{r_{p}(k)}{p^{m}}
$$

for both $k=q$ and $k=q+1$. Corollary 3.7 implies that

$$
r_{p}(q) \leq r_{p}(q-1)\left(1-\frac{1}{p}\right)
$$

therefore

$$
P(q) \leq P(q-1) \prod_{p \leq q-1}\left(1-\frac{1}{p}\right)
$$

By Mertens' theorem

$$
\prod_{p \leq k}\left(1-\frac{1}{p}\right) \sim \frac{e^{-\gamma}}{\log k},
$$

where $\gamma$ is Euler's constant, hence the two probabilities $P(q-1)$ and $P(q)$ will tend to differ by at least a factor which is proportional to $\log q$. Thus, we expect the gaps between $g(q-1)$ and $g(q)$ to increase significantly for large primes $q$.

\section{IMPLEMENTATION}

MSSU utilizes 32 VLSI chips operating in parallel, each of which implements an electronic sieve device performing at a rate of 192 million trials per second. Each individual chip supports the moduli 16, 9, 25, and 49, as well as the next 26 primes 53 through 113 in hardware.

For fixed $k$, the values of all the admissible residues in the sieving problem for $g(k)$ are precomputed in software and passed to MSSU. MSSU then optimizes this information as described below to best fit its hardware. An on-line filter checks each value $n$ which satisfies (2.3) for condition (2.4). The computation terminates as soon as such a value $n$ is let through by the filter, this value being $g(k)$.

Since many of the required moduli $p^{m}$ are not available in hardware, a congruence $\left(\bmod p^{m}\right)$ may be reduced to a congruence $\left(\bmod p^{l}\right)$ where $l<m$. The residues in $B_{m-1}$ are then mapped or folded onto a possibly smaller set of residues $\left(\bmod p^{l}\right)$. The congruences "lost" in the process of residue folding are implemented in software using an off-line filter that screens out "false" solutions. This does not slow down the sieving process, as the number of false solutions is sufficiently small to avoid a bottleneck.

MSSU further optimizes the computation by partitioning congruences. A subset of moduli $\left\{m_{1}, m_{2}, \ldots, m_{g}\right\}$ is selected, and for each modulus $m_{j}$, a subset $\left\{s_{j 1}, s_{j 2}, \ldots, s_{j l_{j}}\right\}$ of the corresponding admissible residues is chosen $(j=$ $1,2, \ldots, g)$. The set of congruences

$$
x \equiv s_{j 1}, s_{j 2}, \ldots, s_{j l_{j}} \quad\left(\bmod m_{j}\right) \quad(j=1,2, \ldots, g)
$$


is combined into a single congruence

$$
x \equiv S_{1}, S_{2}, \ldots, S_{l} \quad(\bmod M)
$$

where $M=m_{1} m_{2} \cdots m_{g}$ and the $S_{i},(i=1,2, \ldots, l)$ are obtained using the Chinese Remainder Theorem. If the set of congruences (5.1) is selected such that $l$ in (5.2) satisfies $l \leq 32$, then each residue $S_{i}$ in (5.2) can be assigned to a different sieve chip. The $i$-th chip now sieves on $M x+S_{i}$ rather than $x$, which results in a speedup of a factor $M$ in the computation. Therefore, the congruences (5.1) should be selected among the many different partitions in such a way that $M$ is maximal. Clearly, congruences with few admissible residues (single residue congruences in particular) and large moduli are most desirable. The number of choices is further increased when partitioning is combined with residue folding, since for partitioning purposes, a reduced modulus $m_{j}=p^{l}$ in (5.1) need not actually be supported by the underlying hardware. Fortunately, the total number of congruences is sufficiently small to make an exhaustive search for the optimal combination of residue folding and congruence partitioning computationally feasible.

We conclude this section with a comment on the speed-up suggested in [1] in the case where $k+1$ is composite. In this case, each solution candidate $n$ for $g(k)$ satisfies $n \equiv-1(\bmod k+1)$, so one can sieve on $(n+1) /(k+1)$ rather than $n$ and speed up the process by a factor of $k+1$. The MSSU algorithm achieves essentially the same speed-up as follows. For each prime divisor $p$ of $k+1$, modulus $p^{m}$ is folded onto modulus $p^{\alpha}$ where $\alpha$ is the largest exponent such that $p^{\alpha} \mid k+1$. This results in a single residue congruence $n \equiv-1\left(\bmod p^{\alpha}\right)$ (see the proof of Lemma 2 in [1]). Combining these congruences for all primes dividing $k+1$ yields a single residue congruence $n \equiv-1 \equiv k(\bmod k+1)$.

We recomputed $g(k)$ for all $k \leq 140$ and found an error in the table given in [1] for $k=138$. The correct value is $g(k)=601242167764223$. We also computed $g(k)$ for $141 \leq k \leq 200$. A table of these values can be found at the end of the paper. To show the enormous increase in speed of MSSU versus OASiS, the device used for the computations in [1], we point out that OASiS required 11 days 11 hours for computing $g(139)$, whereas MSSU achieved this task in a mere 4 minutes (including time to load and verify the problem).

Sieving rates varied greatly for various values of $k$. The fastest sieving rate occurred for $k=199$ with a hardware count rate of $7.5 \times 10^{15}$ per second, requiring less than 20 hours to compute $g(199)$. One of the more difficult values of $k$ to compute was 198 with a hardware sieving rate of $3.3 \times 10^{13}$. This would have taken more than 50 days to compute using only 32 sieve chips. However, we were able to re-partition the problem into 5 subproblems requiring 24 sieve chips each and were able to verify a solution in under 10 days. Due to the very low rate at which solution candidates were generated, solution filtering had a negligible effect on the the observed sieving rate. Surprisingly, even with the introduction of false solutions by residue folding, and optimizing out many of the sparse congruences using partitioning, sieving proceeded at essentially the maximum theoretical hardware sieving rate. Using a 6-way partitioning, it took approximately 30 days to compute the largest value found, $g(200)$, which is a 23 digit number.

In the table of values of $g(k)$, the digits of $g(k)$ are written in groups of at most ten to facilitate reading. Prime values of $k$ are given in bold type.

The authors wish to thank the referee for several helpful suggestions. 


\begin{tabular}{|c|r|}
\hline$k$ & \multicolumn{1}{|c|}{$g(k)$} \\
\hline 135 & 3157756005623 \\
136 & 4138898693368 \\
$\mathbf{1 3 7}$ & 951598054985213 \\
138 & 601242167764223 \\
$\mathbf{1 3 9}$ & 25972027636644319 \\
\hline \hline 140 & 9089854222866845 \\
141 & 63331523816662671 \\
142 & 19904656320115423 \\
143 & 15422895461804543 \\
144 & 1397193586455769 \\
145 & 3395156674599871 \\
146 & 175095016485374 \\
147 & 725311731192223 \\
148 & 11808400809148 \\
$\mathbf{1 4 9}$ & 5423945342959799 \\
\hline \hline 150 & 473138520098551 \\
$\mathbf{1 5 1}$ & 32589899217872863 \\
152 & 155497967465547419 \\
153 & 535184995256751839 \\
154 & 178646907528990874 \\
155 & 41297984000013467 \\
156 & 25272334970944959 \\
$\mathbf{1 5 7}$ & 1041308297102375167 \\
158 & 47025660758882783 \\
159 & 64855518266246559 \\
\hline \hline 160 & 16647456280932287 \\
161 & 3421590108339941 \\
162 & 202129337635322 \\
$\mathbf{1 6 3}$ & 51881272225707439 \\
164 & 146647261829992439 \\
165 & 98652274401898671 \\
166 & 3475847868933047 \\
$\mathbf{1 6 7}$ & 2773085564165092343 \\
168 & 486692232365306798 \\
169 & 726980979380669099 \\
\hline
\end{tabular}

\begin{tabular}{|c|r|}
\hline$k$ & \multicolumn{1}{|c|}{$g(k)$} \\
\hline 170 & 59428415007516671 \\
171 & 284965949074228671 \\
172 & 112232065794463997 \\
$\mathbf{1 7 3}$ & 1381753116390427373 \\
174 & 110577336695616174 \\
175 & 1944092194361247743 \\
176 & 226255303912072703 \\
177 & 192465238561441207 \\
178 & 6844809280136434 \\
$\mathbf{1 7 9}$ & 907874197930300859 \\
\hline \hline 180 & 439763016255983614 \\
$\mathbf{1 8 1}$ & 283361501701232528573 \\
182 & 28988838634918997183 \\
183 & 53516247056143575999 \\
184 & 26626878448827721469 \\
185 & 37136036550263266493 \\
186 & 142741579946200597438 \\
187 & 2208849912824359867 \\
188 & 1271981065611178943 \\
189 & 2956298053153332989 \\
\hline \hline 190 & 455652232192890367 \\
$\mathbf{1 9 1}$ & 9396883214719852991 \\
192 & 1063650724436901873 \\
$\mathbf{1 9 3}$ & 435251419728230720249 \\
194 & 126387435883753706219 \\
195 & 26325912161870817495 \\
196 & 781516664215365373 \\
$\mathbf{1 9 7}$ & 427960977126350089949 \\
198 & 135339364603654686198 \\
$\mathbf{1 9 9}$ & 403168868867096129999 \\
\hline \hline 200 & 52087838892710191382732 \\
\hline
\end{tabular}

\section{REFERENCES}

1. R. Scheidler and H. C. Williams, A method of tabulating the number theoretic function $g(k)$, Math. Comp. 59 (1992), 251-257. MR 92k:11146

2. E. F. Ecklund, P. Erdös and J. L. Selfridge, A new function associated with the prime factors of $\left(\begin{array}{c}n \\ k\end{array}\right)$, Math. Comp. 28 (1974), 647-649. MR 49:2501

3. P. Erdös, C. B. Lacampagne and J. L. Selfridge, Estimates of the least prime factor of a binomial coefficient, Math. Comp. 61 (1993), 215-224. MR 93k:11013

4. R. F. Lukes, C. D. Patterson and H. C. Williams, Numerical Sieving Devices: Their History and Some Applications, Nieuw Archiv voor Wiskunde 13, ser. 4, no. 1 (1995), 113-139. MR 96m:11082 
5. A. Granville and O. Ramaré, Explicit bounds on exponential sums and the scarcity of squarefree binomial coefficients, Mathematika, 43 (1996), 73-107. CMP 96:16

6. L. E. Dickson, History of the Theory of Numbers, vol. 1, Chelsea, New York, 1966. MR 39:6807a

Department of Computer Science, University of Manitoba, Winnipeg, Manitoba, CANADA R3T 2N2

E-mail address: rflukes@cs.umanitoba.ca

Department of Mathematical Sciences, University of Delaware, Newark, Delaware 19716

E-mail address: scheidle@math.udel.edu

Department of Computer Science, University of Manitoba, Winnipeg, Manitoba, Canada R3T 2N2

E-mail address: hugh_williams@csmail.cs.umanitoba.ca 